\title{
OBJECT-LOCATING VIA OBJECT RECOGNITION AND INDOOR POSITIONING
}

\author{
Chuan-Kai Yang and Hsuan Chin \\ National Taiwan University of Science and Technology, Taipei, Taiwan
}

\begin{abstract}
Object locating has been a problem in our daily lives, and some techniques have been proposed to address this issue. However, there are still some drawbacks with previous methods. This paper proposes to use two techniques to make object locating easier through object recognition and indoor positioning, so that not only a picture containing the desired object can be shown to help recall where the object was placed, but also the location or room can be shown to help guide where to retrieve the desired object.
\end{abstract}

\section{KEYWORDS}

Object Locating, Indoor Positioning, Object Recognition

\section{INTRODUCTION}

Modern people tend to become more forgetful as there are too many things to remember, and this is especially true when we tried to recall where a particular object was finally placed. The problem, termed as object locating, has become an issue that the paper tries to address. Existing techniques mostly resort to particular devices or multiple cameras. However, the involved hardware either causes higher cost or lacks particular orientation or positioning information, thus making the corresponding solutions less attractive. We propose to make use of a commonly available mobile phone to develop two versions for locating a desired object. The first version of our system takes a picture of a desired object, sends the picture for object recognition, and stores the returned recognized object name with the picture so that later on such a picture can be retrieved through a voice command to help recall where the object was finally put. The second version, aims to further improve the first one, by taking a picture of the object attached with a specially designed paper tag so that we could infer the relative distance and orientation of the object with respect to the mobile phone. Together with the use of some indoor positioning technique to record the position of the mobile phone when taking the picture, we later on could guide a user to the location/room where the object was placed last time. The rest of the paper is organized as follows. Section 2 reviews work related to this paper. Section 3 describes how the first version of our system is implemented. Section 4 details how we based on the first version to further improve it to develop our second version. Section 5 presents some of our results. Section 6 concludes this paper and hints for possible future directions.

\section{RELATED WORK}

Regarding object locating, there exist several systems, as surveyed by Koyuncu and Yang, (Koyuncu and Yang, 2010), while most of them applied some specific hardware to help locate desired objects. Another representative work, done by Frank et al. (Frank et al., 2007), is also to make use of RFID/Bluetooth technique for object locating. Although the work is quite complete, the lack of orientation information and limitation of the sensing range for using a mobile phone as a signal reader has made the system more complicated. To resolve these issues, as mentioned previously, this work applies basically two techniques, i.e., object recognition and indoor positioning, to help address the object locating problem. We therefore also briefly review these two fields of study respectively. 
In terms of object recognition, there are many related studies using robots to identify objects. In 2016, Rafsan et al. (Rafsan et al., 2016) proposed a human-robot interaction framework. Robots can detect specific objects at home according to the needs of users. They use Haar classifiers to identify them. For Objects at home, they can distinguish specific objects based on their colors, sizes, or positional information. In addition to using robots, cameras can also be used to identify objects. In 2015, Liaquat et al. (Liaquat et al., 2015) proposed a single camera-based depth estimation technique. After detecting different objects in the room, they calculate their areas, and then estimate the distance between the camera and the object based on the training data and a polynomial curve fitting method. In 2012, Mustafah et al. (Mustafah et al., 2012) proposed a more accurate measurement of the distance and size of objects in the "field of view" using a stereo vision system of two cameras.

In terms of indoor positioning, since the GPS positioning system cannot be used indoors, other positioning technologies have been proposed, such as iBeacon, geomagnetic positioning, Bluetooth, and wifi.iBeacon: In 2017, Dalkılıç et al. (Dalkılıç et al., 2017) tested the accuracy of the distance between two devices communicating via iBeacon technology. The results of the study indicate that iBeacon technology is suitable for indoor positioning systems where accuracy is not critical. The placement of iBeacons and environmental conditions are important for obtaining more accurate distance estimates. Regarding geomagnetic positioning, in 2014, Pritt (Pritt, 2014) investigated the possibility of using indoor geomagnetism to enhance the accuracy of wifi indoor positioning. The results show that the combination of geomagnetic and Wi-Fi fingerprint recognition can improve the accuracy of indoor positioning. Regarding Bluetooth, in 2018 Satan (Satan, 2018) used a Bluetooth beacon to transmit RF signals for distance calculation. Based on the distance from the beacon, the user's position is estimated and Dijkstra's shortest path algorithm is used for route planning.

\section{OBJECT INFORMATION COLLECTION AND RETRIEVAL}

\subsection{System Flow for Object Information Collection}

The object detection and positioning process is divided into three phases:

(1) First, take a picture of the desired object.

(2) Use Tensorflow Mobile to detect/recognize the object on the mobile phone, and also detect the marker on the object.

(3) Combine the direction sensor of the mobile phone itself to know the positioning coordinates of the object relative to the mobile phone.

\subsection{Object Recognition}

Google's object detection API - Tensorflow is an open source software library for high performance numerical computation. Its flexible architecture allows easy deployment of computation across a variety of platforms (CPUs, GPUs, TPUs), and from desktops to clusters of servers to mobile and edge devices. If we use/download an engine that can recognize the desired object already, then we don't need to perform any training. However, if the object in question cannot be recognized, then we need to train the engine ourselves.

\subsection{Calculation of Distance}

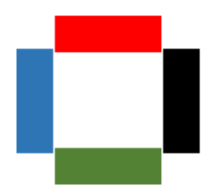

Figure 1 
In order to quickly and easily know the distance of the object, we designed a marker as shown in Figure 1 to be attached on the object. As this marker is with a fixed size, its distance, as well as its orientation, to the camera, can be calculated according to the pin-hole camera formula.

\subsection{Calculation of Orientation}

Once we know the distance of the object, denoted as $r$ in Figure 2, and then we can use the direction sensor of the mobile phone to obtain the current directional angle of the mobile phone, and the trigonometric function can be used to obtain the $\mathrm{X}, \mathrm{Y}$ coordinates of the object relative to the mobile phone (user).

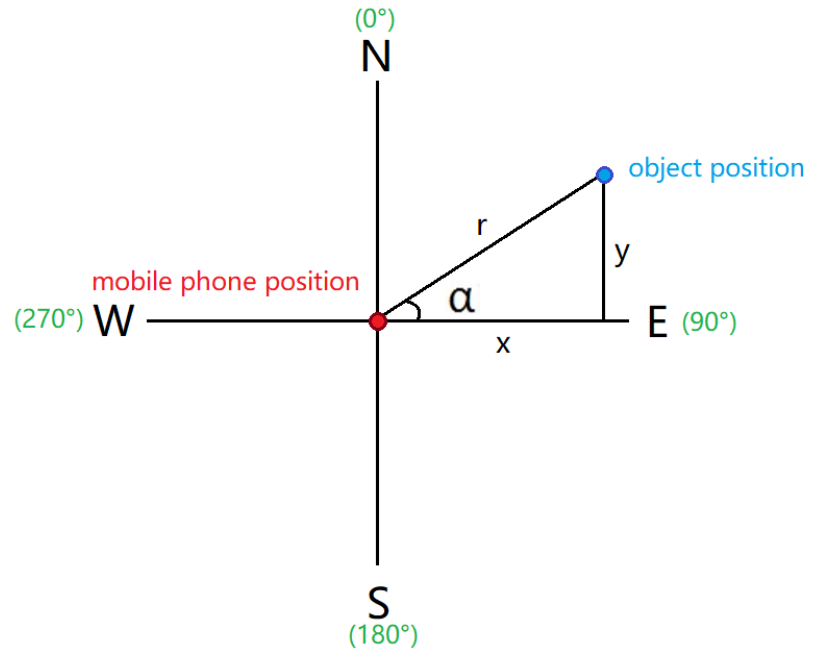

Figure 2

\subsection{Voice Retrieval}

The image, name, distance, orientation and coordinate information of the object are stored in the database. Users can use voice command to retrieve to find the objects they are looking for.

\section{INDOOR POSITIONING}

\subsection{Geomagnetic Indoor Positioning Technology}

The Earth itself is a giant magnet that forms a magnetic field between the geographic north and south. However, the earth's magnetic field is disturbed by metal objects, which creates a unique magnetic force map inside each building, can cause the different magnetic field differences at various points in the room for indoor positioning. The advantage is that we can use existing equipment without deploying other hardware infrastructure. The disadvantage is that the magnetic field changes must be actually collected at various locations in the room, and the preparation process is troublesome.

\subsection{Indoor Atlas}

IndoorAtlas is a Finnish start-up that offers a geomagnetic indoor positioning platform. It is expected to provide users with indoor positioning services with an accuracy of 1 meter by effectively utilizing the functions of the smart phone built-in magnetometer and through its cloud-based geomagnetic map service.

IndoorAtlas Business Director Daniel Patton said, "This technology is very simple, because modern buildings have a unique magnetic field feature." The structure and materials of the building interact with the 
Earth's magnetic field, creating a unique magnetic map on each floor. Once this magnetic map is recorded and stored in the cloud, it can be used to accurately find and track the individual's position indoors.

\subsection{Indoor Positioning Process}

The process of indoor positioning is divided into 5 steps:

(1) First upload the indoor floor plan to the IndoorAtlas platform.

(2) Using the MapCreator app provided by Indooratlas to actually visit the entire indoor environment and collect geomagnetic data from various locations in the room.

(3) The collected geomagnetic data will be uploaded to the IndoorAtlas platform and converted into different X, Y coordinates according to the change of geomagnetism.

(4) Then according to the difference of geomagnetism, we can use the API provided by IndoorAtlas to get the $\mathrm{X}, \mathrm{Y}$ coordinates of the current position.

(5) Finally, the technique of walking and painting can be implemented according to the user's movement. And in such a manner a user can be aware where he/she is and as long as the location (room) of the object is known, this can be used to guide the user to the reach where the object is placed.

\section{RESULTS}

Table 1. Development Environment

\begin{tabular}{|l|l|}
\hline Development Platform & Android Studio \\
\hline Program language & Java \\
\hline Use Packages & (1) Object detection library : \\
& library 'org.tensorflow:tensorflow-android:+' \\
& (2) Indoor positioning package : \\
& Package 'com.indooratlas.android.sdk' \\
\hline Phone specifications & Sony Xperia Z3+ \\
\hline
\end{tabular}

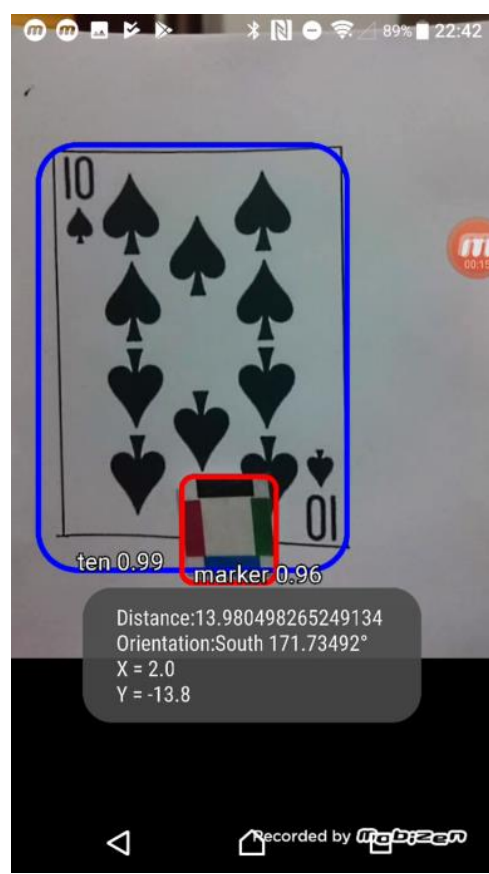

(a)

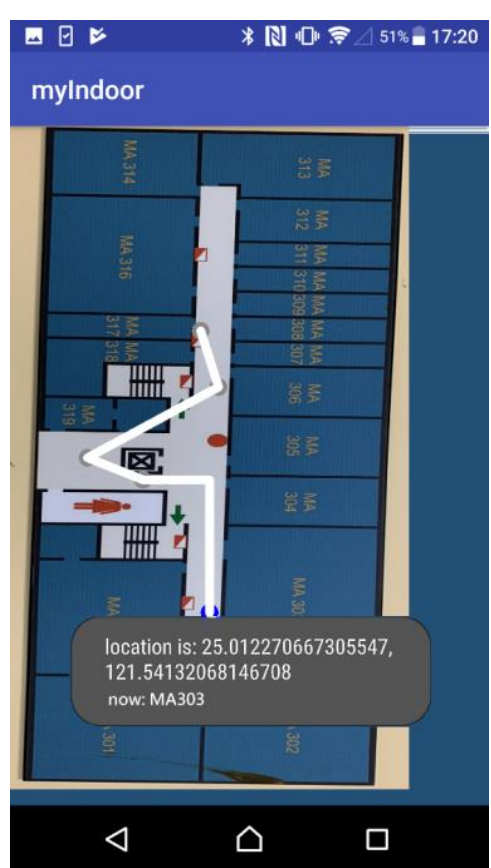

(b)

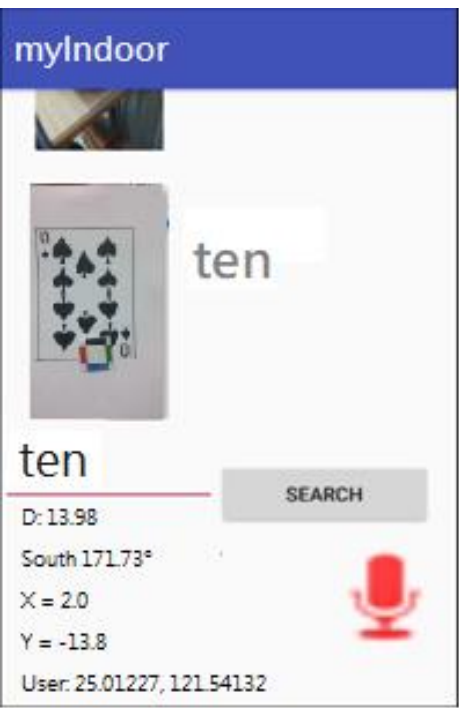

(c)

Figure 3 
Table 1 shows our development environment. The testing environment is the 3rd Floor in our school, and the Figure 3(a) shows the object detection result, which recognizes the playing cards 10 and markers in the picture, and shows that the distance from the mobile phone (user) is $13.98 \mathrm{~cm}$, located at $171.73^{\circ}$ in the south, and the coordinate relative to the mobile phone (user) is $(2.0,-13.8)$.

Figure 3(b) shows the result of the painting while walking. The gray point is the location where the user visited. The blue point is the user's current position, and the white line segment is the line segment formed by walking. Figure 3(c) shows the result of the voice retrieval. A user can press the red microphone icon to enter the name of the item by voice. He/she then presses the search button to display the photo containing the desired object.

\section{CONCLUSION AND FUTURE WORK}

We have proposed two versions for object locating. The first one makes use of object detection by taking a picture of a desired object and recording the recognized name into a database so that later on the associated picture can be retrieved through a voice command to help recall where the object was placed last time. The second version improves the first one by incorporating the relative distance and orientation of the object with respect to the image-capturing mobile phone, together with the location of the mobile phone and a third-party indoor positioning system to guide the user to where the object was finally put.

There are still some limitations in our current system. First, to make sure that our system works, a user needs to take a picture of an object each time it is moved to a new place. Second, even though an object may stay where it is, it is still possible that some other object is placed in front of it to prevent it from being seen. Third, currently our system can only show a user where the object was placed without actively guiding the user toward the object. In the future, to address the first two issues, one or more surveillance cameras could be installed to keep track of how and where the objects are displaced. To address the third issue, we believe some other functionality of third-party indoor positioning tool can be employed to achieve this.

\section{ACKNOWLEDGEMENT}

This work was supported in part by the Ministry of Science and Technology of Taiwan under the grants MOST 104-2221-E-011-083-MY2, MOST 105-2218-E-011-005, MOST 105-2218-E-001-001, MOST 106-3114-E-011-003, and MOST 106-2221-E-011-148-MY3.

\section{REFERENCES}

Dalkılıç et al., 2017. An Analysis of the Positioning Accuracy of iBeacon Technology in Indoor Environments, International Conference on Computer Science and Engineering (UBMK), pp. 549-553.

Pritt, 2014. Indoor Navigation with use of Geomagnetic Anomalies, IEEE Geoscience and Remote Sensing Symposium, pp. 1859-1862.

Satan, 2018. Bluetooth-based Indoor Navigation Mobile System, 19th International Carpathian Control Conference (ICCC), pp. 332-337.

Rafsan et al., 2016. Design a Human-Robot Interaction Framework to Detect Household Objects, International Conference on Informatics, Electronics and Vision (ICIEV), pp. 973-978.

Liaquat et al., 2015. Object Detection and Depth Estimation of Real World Objects using Single Camera, Fourth International Conference on Aerospace Science and Engineering (ICASE), pp. 1-4.

Mustafah et al., 2012. Stereo vision images processing for real-time object distance and size measurements, 2012 International Conference on Computer and Communication Engineering (ICCCE), pp. 659-663.

Frank et al., 2007. Objects Calling Home: Locating Objects Using Mobile Phones, Pervasive Computing. Pervasive 2007.

Koyuncu and Yang, 2010. A Survey of Indoor Positioning and Object Locating Systems, International Journal of Computer Science and Network Security, Vol. 10, No. 5, pp. 121-128. 